\title{
A meta-analysis of the association between preoperative magnetic resonance imaging and surgical outcomes in newly diagnosed breast cancer
}

\author{
Li-Li Xie, Zhi-Hong Xu, Deng-Hui Wei, Chun-Sen Xu
}

Fujian Medical University Union Hospital, Fuzhou, Fujian Province, China

Submitted: 12 September 2019; Accepted: 24 April 2020

Online publication: 10 April 2021

Arch Med Sci

DOI: https://doi.org/10.5114/aoms/120995

Copyright $\odot 2021$ Termedia \& Banach

\begin{abstract}
Introduction: The use of preoperative magnetic resonance imaging (MRI) in newly diagnosed breast cancer has been increasing. However, the value of MRI applied to women with newly diagnosed breast cancer remains controversial. Therefore, this study focused on the association between preoperative MRI and surgical outcomes, which include mastectomy rate, breast conservation rate, and re-excision rate.

Material and methods: A systematic literature search was performed by the Wanfang, VIP (Chinese biomedical databases), PubMed, Cochrane Library, and Embase, databases. The keywords were "breast cancer", "magnetic resonance imaging", "mastectomy rate", "re-excision rate", and "breast conservation rate". A random-effects model was used to estimate the proportion of women with various outcomes in the MRI group versus the non-MRI group. The odds ratio (OR) for each surgical outcome was calculated.

Results: 4 RCTs (randomized controlled trials) met the eligibility criteria that included a total of 2312 patients with breast cancer. The analysis results of the MRI group versus the non-MRI group were as follows: mastectomy rate of $15 \%$ versus $10 \%, O R=2.01,95 \% \mathrm{Cl}: 0.79-5.11, p=0.14$; breast conservation rate $84 \%$ versus $89 \%, \mathrm{OR}=0.58,95 \% \mathrm{Cl}: 0.29-1.17, p=0.13$; re-excision rate $17 \%$ versus $19 \%, \mathrm{OR}=0.76,95 \% \mathrm{Cl}: 0.37-1.54, p=0.45$.

Conclusions: There was no significant association between preoperative MRI and surgical outcomes. This study suggested that the use of preoperative MRI should be selective. The results showed that the application of preoperative MRI did not improve the prognosis of breast cancer patients. More randomized trials are needed for further study due to limited randomized trials.
\end{abstract}

Key words: MRI, breast cancer, mastectomy rate, breast conservation rate, re-excision rate, meta-analysis.

\section{Introduction}

Currently, the examinations for breast cancer include breast ultrasonography, mammography, magnetic resonance imaging (MRI), and elastography. Breast ultrasonography and mammography are the most common assessments for breast diseases [1]. Imaging possibilities and quantitative information of breast are accessible by elastography, which is an imaging technique developed over the last 20 years [2-4]. Each of them has its advantages, but also certain limitations. Many studies have shown that preoperative MRI can identify additional foci of cancer with

\author{
Corresponding author: \\ Chun-Sen Xu \\ Fujian Medical University \\ Union Hospital \\ Fuzhou, 350001 \\ Fujian Province, China \\ E-mail: \\ xuchunsen909@163.com
}


occult cancers that are different from conventional examinations. Therefore, the use of MRI in clinical practice has become more popular $[5,6]$. It has been well established that MRI detects unsuspected sites of cancer, for example, in the ipsilateral breast in $16 \%$ of women. It also identifies occult cancer in the contralateral breast, for example, in $3 \%$ to $5 \%$ of women with newly diagnosed breast cancer $[7,8]$. These studies have also shown that most of these lesions will be malignant. However, the benefits of MRI as a preoperative assessment tool remain controversial. Some studies have shown that MRI can increase the rate of mastectomy [9] and some have shown that MRI can reduce the rate of breast re-excision [10], so MRI can improve the surgical outcomes in breast cancer patients. However, some other articles reported no significant association between MRI and surgical outcomes, which included mastectomy rate and re-excision rate [11-13].

A meta-analysis conducted by Houssami et al. in 2017 analyzed the association between preoperative MRI and surgical outcomes, which included 16 retrospective studies and 3 RCTs (randomized controlled trials) [14]. The analysis showed that preoperative MRI increased the rate of breast mastectomy. However, the study also had many limitations: (1) Most of the studies included in the analysis were retrospective studies, while only 3 were RCTs. (2) The difference between the sample sizes was too large; the maximum sample size was 53015 cases and the minimum was 149 cases. This difference in sample size may have affected the analysis results. (3) The heterogeneity of 7 analyses was more than 50\%, while the author did not explore the source of heterogeneity. (4) The article failed to perform a sensitivity analysis, which may have led to the uncertainty of the results [15]. Therefore, it is necessary to perform a meta-analysis to examine the surgical outcomes of preoperative MRI in breast cancer patients, with a focus on RCTs to increase the standard of the evidence level. The purpose of this study is to determine the effect of MRI on standard preoperative results in assessing short-term surgical outcomes, including mastectomy rate, breast conservation rate, and re-excision rate.

\section{Material and methods}

\section{Literature research strategy}

A systematic literature search was performed using the Wanfang, VIP (Chinese biomedical databases), PubMed, Cochrane Library, Embase, and Vip databases from their establishment to December 2018. To identify the studies that reported surgical outcomes of preoperative MRI in patients diagnosed with breast cancer, we used the following keywords: "breast cancer", "magnetic resonance imaging", "mastectomy rate", "re-excision rate", and "breast conservation rate". The data for mastectomy rate, breast conservation rate, and re-excision rate were extracted from the two groups, the MRI group versus the non-MRI group.

\section{Inclusion criteria}

The selected studies were required to fulfill a set of inclusion criteria as follows: prospective randomized clinical trials; breast cancer had to be diagnosed by pathological examination, including invasive carcinoma, intraductal carcinoma in situ; articles published up to December 2018; English or Chinese articles; the sample size was greater than or equal to 100 cases; studies that provided direct or indirect access to the data of mastectomy rate, breast conservation rate, and re-excision rate.

\section{Exclusion criteria}

The selected studies were required to fulfill a set of exclusion criteria as follows: articles of abstracts, reviews, retrospective studies, or case reports; studies without a control group; studies with a sample size that was less than 100 cases; for multiple articles published by the same authors, we selected the one with the best quality; studies with incomplete data.

\section{Data extraction}

Two authors independently reviewed the abstracts from the literature search and identified the potentially relevant articles. Two authors extracted the following data: publication details, study population and time frame, the titles of the articles, the first author, study design, total number of patients with breast cancer, average age of study subjects, mastectomy rates, breast conservation rates, and re-excision rates. To assess the quality of the studies, the following information was also extracted: study design (whether randomization was used and whether the study was prospective or retrospective). Data extraction was consistent between the two reviewers. When the data on the surgical outcomes were reported but clarification was needed, the authors of the study were contacted to confirm the data or to obtain clarification. To avoid overlapping patient groups and where studies reported on the same patients in more than one publication, the most informative and/or most recent article was included.

\section{Quality assessment}

We assessed each article's risk of bias, with the tool recommended by the Cochrane Collaboration. The tool consists of a description and a judg- 
ment for the following domains in a "risk of bias table": random sequence generation, allocation concealment, blinding of participants and personnel, blinding of outcome assessments, incomplete outcome data, selective reporting, and other bias. Each domain was judged as "low risk", "high risk" or "unclear" for each article.

\section{Statistical analysis}

The odds ratios (OR) of each outcome with a 95\% confidence interval $(\mathrm{Cl})$ were calculated for each study. Random-effects logistic meta-regression was used to model the proportion of subjects with surgical outcomes. Statistical heterogeneity among the studies was evaluated with the Q test and $l^{2}$ statistic. Meanwhile, a funnel plot was used to assess reporting bias. If the majority of studies were on the top of the inverted funnel, with bilateral symmetry on the funnel plot, then it indicated that there was no apparent reporting bias. Review Manager 5.3 (RevMan, Version 5.3 for Windows, Nordic Cochrane Centre, 2008) was used for the meta-analysis.

\section{Results}

\section{Characteristics of the studies}

Our search strategy identified 513 articles; of these, 4 studies $[10-12,16]$ met the eligibility criteria. All of them were RCTs, including 2312 newly diagnosed breast cancer patients. The study flow diagram is shown in Figure 1. The characteristics of each study are summarized in Table I.

\section{Statistical heterogeneity}

To precisely assess the heterogeneity of this study, the $\mathrm{Q}$ test and $l^{2}$ statistics were calculated separately for the mastectomy rate, breast conservation rate, and re-excision rate and the sensitivity analysis was conducted after the $\mathrm{Q}$ test and $I^{2}$ statistic. Mastectomy rate: the result of the mastectomy rate was: $\operatorname{tau}^{2}=0.72 ; \chi^{2}=21.09, \mathrm{df}=3$, $p=0.0001 ; I^{2}=86 \%$; it showed that the heterogeneity of these trials was substantial and the sensitivity analysis showed that the trial "Turnbull 2010"

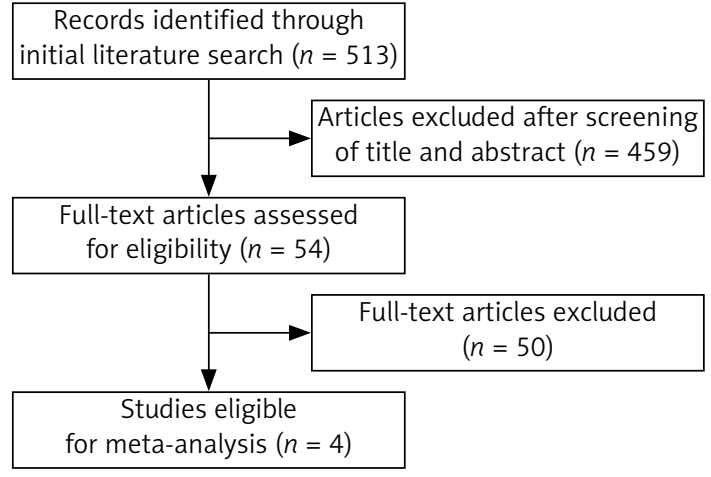

Figure 1. Study flow diagram

was the reason for the heterogeneity. If we had excluded this trial, there would have been no heterogeneity present in the overall analysis $\left(\operatorname{tau}^{2}=0.03\right.$; $\left.\chi^{2}=2.41, \mathrm{df}=2, p=0.30 ; I^{2}=17 \%\right)$. Breast conservation rate: the result of the breast conservation rate was: $\operatorname{tau}^{2}=0.36 ; \chi^{2}=14.31, \mathrm{df}=3, p=0.003$; $R^{2}=79 \%$; it showed that the heterogeneity of these trials was substantial and the sensitivity analysis showed that trial "Turnbull 2010" was the reason for the heterogeneity. If we had excluded this trial, there would have been no heterogeneity present in the overall analysis $\left(\mathrm{tau}^{2}=0.03 ; \chi^{2}=2.46\right.$, $\left.\mathrm{df}=2, p=0.29 ; l^{2}=19 \%\right)$. Re-excision rate: the result of the re-excision rate was: $\operatorname{tau}^{2}=0.40$; $\chi^{2}=14.84, \mathrm{df}=3, p=0.002 ; I^{2}=80 \%$; it showed that the heterogeneity of these trials was also substantial and the sensitivity analysis showed that trial "Gonzalez 2014" was the reason for this heterogeneity. If we had excluded this trial, the heterogeneity would have been significantly reduced $\left(\operatorname{tau}^{2}=0.16 ; \chi^{2}=4.80, \mathrm{df}=2, p=0.09,1^{2}=58 \%\right)$. Heterogeneity was substantial for all 3 outcomes, but not all were from the same trial.

\section{Risk of bias}

The results of the risk bias analysis are summarized in Figures 2 and 3. The overall results showed a low risk of bias and the funnel plot suggested that the study bias was not significant (Figures 4-6).

Table I. Summary characteristics of studies of preoperative magnetic resonance imaging included in this meta-analysis

\begin{tabular}{|c|c|c|c|c|c|c|c|c|c|c|c|c|}
\hline \multirow[t]{2}{*}{ Author [Ref.] } & \multirow[t]{2}{*}{ Year } & \multirow[t]{2}{*}{ Style } & \multicolumn{2}{|c|}{$\begin{array}{c}\text { Patient } \\
\text { number }(n)\end{array}$} & \multicolumn{2}{|c|}{$\begin{array}{l}\text { Median or mean } \\
\text { age* (years) }^{*}\end{array}$} & \multicolumn{2}{|c|}{$\begin{array}{c}\text { Mastectomy } \\
(n)\end{array}$} & \multicolumn{2}{|c|}{$\begin{array}{c}\text { Breast } \\
\text { conservation }(n)\end{array}$} & \multicolumn{2}{|c|}{$\begin{array}{l}\text { Re-excision } \\
\text { (n) }\end{array}$} \\
\hline & & & MRI & no-MRI & MRI & no-MRI & MRI & no-MRI & MRI & no- MRI & MRI & no- MRI \\
\hline Brück [9] & 2017 & $\mathrm{RCT}$ & 50 & 50 & 61 & 61 & 6 & 2 & 44 & 48 & 7 & 12 \\
\hline Gonzalez [7] & 2014 & $\mathrm{RCT}$ & 220 & 220 & 46 & 46 & 86 & 75 & 131 & 143 & 11 & 33 \\
\hline Peters [13] & 2011 & $\mathrm{RCT}$ & 74 & 75 & $55.1^{*}$ & $56.1^{*}$ & 23 & 26 & 51 & 49 & 22 & 13 \\
\hline Turnbull [8] & 2010 & $\mathrm{RCT}$ & 816 & 807 & 57 & 57 & 58 & 10 & 750 & 787 & 153 & 156 \\
\hline All & - & - & 1160 & 1152 & - & - & 173 & 113 & 976 & 1027 & 193 & 214 \\
\hline
\end{tabular}

${ }^{\star}$ Age was presented as mean age only in Peters (2011), while others were presented as median age. 


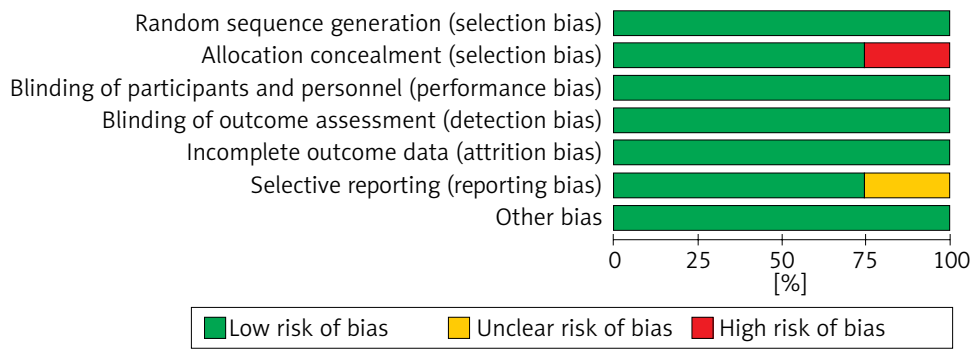

Figure 2. Risk of bias graph

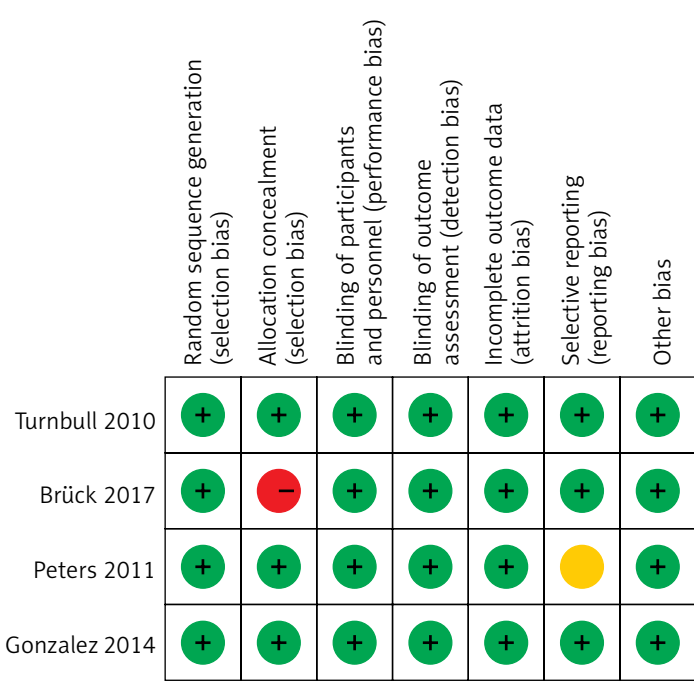

Figure 3. Risk of bias summary

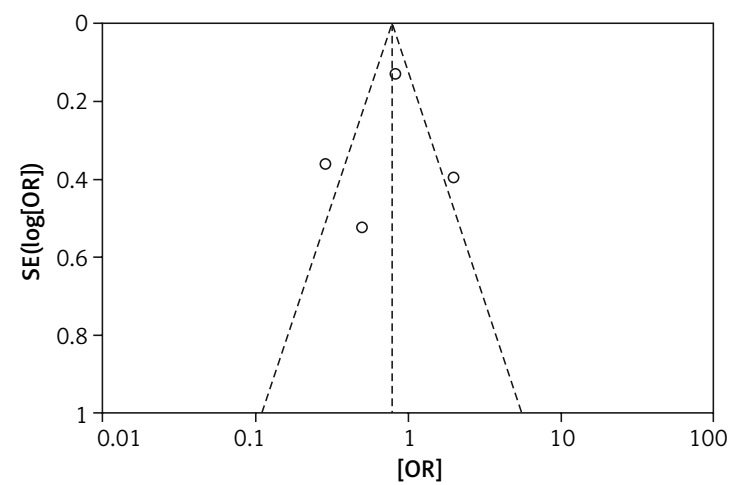

Figure 6. Funnel plot of comparison (re-excision)

\section{Correlation between MRI and surgical outcomes}

The analysis results of the MRI group versus the non-MRI group were as follows. The mastectomy rate of the two groups was $15 \%(173 / 1160)$ versus $10 \%(113 / 1152), \mathrm{OR}=2.01,95 \% \mathrm{Cl}: 0.79-$ $5.11, p=0.14$ (Figure 7). The breast conservation rate of the two groups were $84 \%(976 / 1160)$ versus $89 \%(1027 / 1152), \mathrm{OR}=0.58,95 \% \mathrm{Cl}: 0.29-1.17$, $p=0.13$ (Figure 8 ). The re-excision rates (defined as those patients who were confirmed as

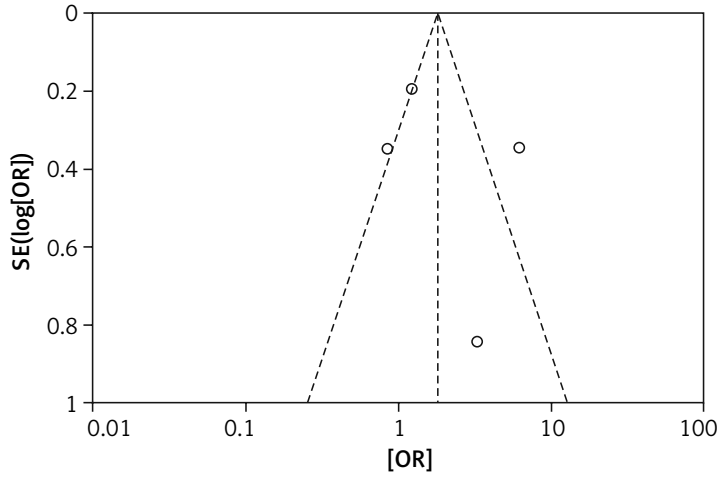

Figure 4. Funnel plot of comparison (mastectomy)

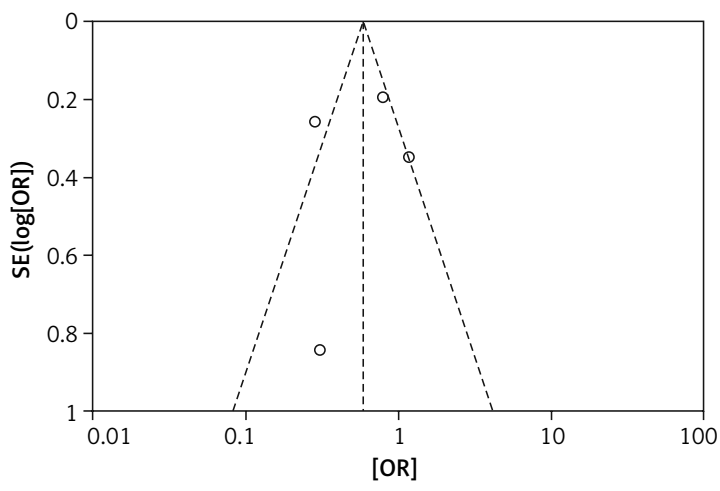

Figure 5. Funnel plot of comparison (breast conservation)

margin positive and who need a second surge) of the two groups were $17 \%(193 / 1160)$ versus 19\% (214/1152), OR =0.76, 95\% Cl: 0.37-1.54, $p=0.45$ (Figure 9), which indicated that there was no significant correlation between preoperative MRI and surgical outcomes that include mastectomy rate, breast conservation rate, and re-excision rate.

\section{Discussion}

The use of preoperative MRI in breast cancer has been increasing in recent years. It has been suggested that MRI could detect unsuspected breast cancers that are not apparent during mammography and ultrasound [17-27]. However, the value of preoperative MRI in newly diagnosed breast cancer remains controversial $[8,28-33]$. This meta-analysis not only studied the 


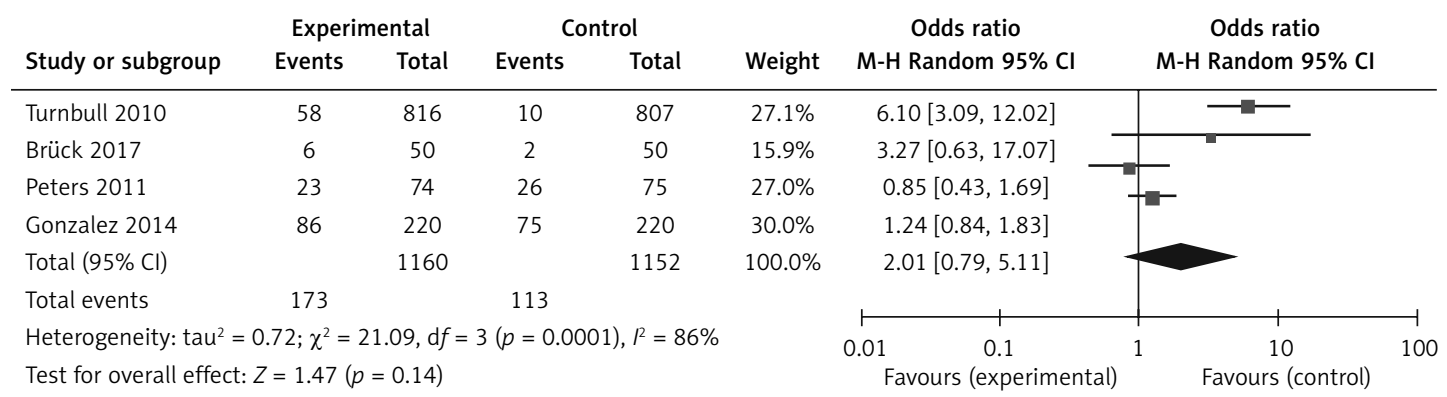

Figure 7. Forest plot of the comparison of efficacy outcome: HR (mastectomy)

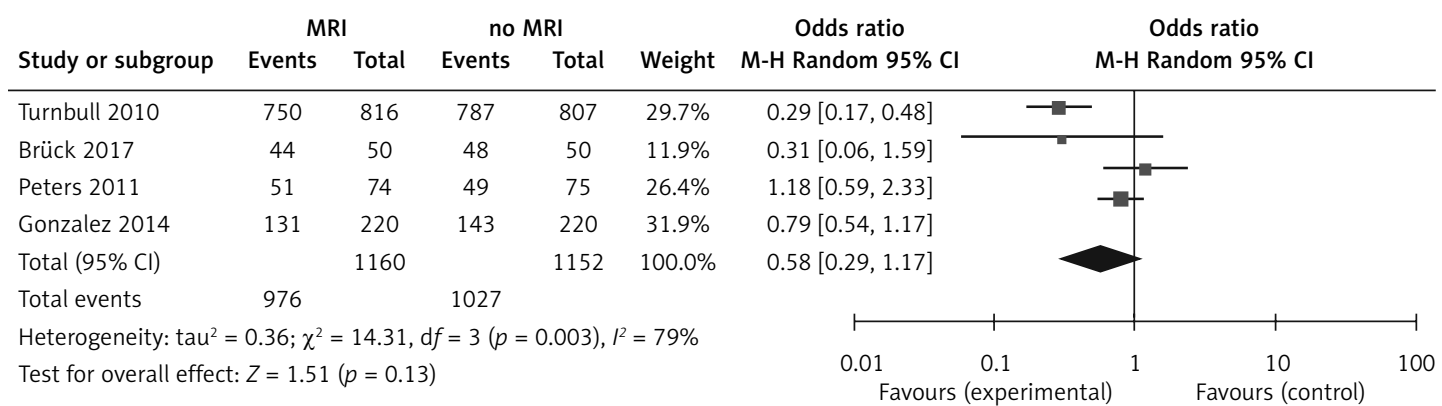

Figure 8. Forest plot of the comparison of efficacy outcome: HR (breast conservation)

\begin{tabular}{|c|c|c|c|c|c|c|c|c|c|}
\hline \multirow[b]{2}{*}{ Study or subgroup } & \multicolumn{2}{|c|}{ MRI } & \multicolumn{2}{|c|}{ no $M R I$} & \multicolumn{2}{|r|}{ Odds ratio } & \multirow{2}{*}{\multicolumn{2}{|c|}{$\begin{array}{c}\text { Odds ratio } \\
\mathrm{M}-\mathrm{H} \text { Random } 95 \% \mathrm{Cl}\end{array}$}} & \\
\hline & Events & Total & Events & Total & Weight & M-H Random $95 \% \mathrm{Cl}$ & & & \\
\hline Brück 2017 & 7 & 50 & 12 & 50 & $19.5 \%$ & $0.52[0.18,1.44]$ & \multirow[t]{2}{*}{$\longrightarrow$} & 7 & \\
\hline Gonzalez 2014 & 11 & 220 & 33 & 220 & 24.9 & $0.30[0.15,1.61]$ & & & \\
\hline Peters 2011 & 22 & 74 & 13 & 75 & $23.7 \%$ & $2.02[0.93,4.39]$ & & & \\
\hline Turnbull 2010 & 153 & 816 & 156 & 807 & $31.9 \%$ & $0.96[0.37,1.54]$ & & & \\
\hline Total $(95 \% \mathrm{Cl})$ & & 1160 & & 1152 & $100.0 \%$ & $0.76[0.37,1.54]$ & & & \\
\hline Total events & \multicolumn{2}{|l|}{193} & \multicolumn{2}{|l|}{214} & & & & & \\
\hline \multicolumn{6}{|c|}{ Heterogeneity: $\tan ^{2}=0.40 ; \chi^{2}=14.84, \mathrm{~d} f=3(p=0.002), I^{2}=80 \%$} & 0.01 & 0.1 & 10 & 100 \\
\hline \multicolumn{6}{|c|}{ TTest for overall effect: $Z=0.76(p=0.45)$} & Favours ( & experimental) & Favours (co & \\
\hline
\end{tabular}

Figure 9. Forest plot of the comparison of efficacy outcome: HR (re-excision)

association between preoperative MRI and surgical outcomes, but also analyzed the effects of preoperative MRI on the surgical outcomes. This study found no statistically significant differences between the two groups in the mastectomy rate, breast conservation rate, and re-excision rate. Also, MRI could increase the medical expenses of breast cancer patients. Therefore, it is reasonable to consider that the benefit of MRI for the prognosis of breast cancer patients is still questionable.

A problem that was found in this study was heterogeneity. In the mastectomy rate analysis and breast conservation rate analysis, the results were all from "Turnbull 2010" [34]. One possible explanations for this heterogeneity is that "Turnbull 2010" had more patients in the proportion that chose breast conservation, rather than mastectomy at the beginning. The mastectomy rate of this study was $4 \%(68 / 1623)$, while other studies had a mastectomy rate of $8 \%$ (8/100), 37\% (161/440), and $33 \%(49 / 149)$. The breast conservation rate of "Turnbull 2010" was 95\% (1537/1623), while in other studies the rates were $92 \%$ (92/100), 62\% (274/440), and 67\% (100/149). "Turnbull 2010" has a significantly higher breast conservation rate than other studies, which may be the source of this heterogeneity. Also, according to the method in the article, mastectomy or breast conservation was chosen depending on the type of the tumor, as well as the patients' preference, and this was supported by the guidelines which suggested that surgeons should take the patient's preference into account. The preference of surgeons and medical centers would affect the patient's choice in their communication about formulating a surgery plan. Also, different cultures, religions, and family opinions would affect the patient's preferences. All of these possibilities could be the reasons for clinical heterogeneity, which may be inevitable if we want to conduct a multiple nation (center) study. As for the heterogeneity of re-excision rate, it mostly came from "Gonzalez 2014" [10]. The mastectomy rate of "Gonzalez 2014" was 37\% (161/440), while other studies had mastectomy rates of $8 \%$ 
(8/100), 33\% (49/149), and 4\% (68/1623). “Gonzalez 2014" included proportionally more mastectomy patients, which could have led to a lower recurrence rate and fewer residual lesions. In this case, there would be a lower re-excision rate. Moreover, environmental and epigenetic factors could affect these results in various ways in different populations from different countries and social strata, which could, for instance, affect the quality of medical service and nutritional factors, which may need further systematic research. Additionally, it was proposed that the following groups would benefit most from preoperative MRI: (1) the histopathological type was invasive lobular cancer or tumor with high malignancy, such as triple negative breast cancer and HER2 positive tumor; (2) young patients, especially those younger than 60 years; (3) multifocal tumor [35].

Despite the heterogeneity, removing the article that caused the heterogeneity did not affect the experiment reaching the same conclusion. Because the stated articles above showed an opposite trend in the analysis, removing them only led to the same conclusion. In this case, we believe this heterogeneity did not affect the reliability of this study. However, with more high-quality trials with a similar criterion, first surgery cases would be a significant help to reach a concrete conclusion.

There were also some good points of this study: All the included studies were prospective randomized controlled studies with greater reliability than retrospective studies and the overall results showed a low risk of bias. Also, the funnel plot suggested that the study bias was not significant.

One of the limitations of this study was that the number of RCT studies was small; only 4 qualifying studies were found within the research deadline. Although one of the studies wrote "random grouping" in the methods section, it did not specify the randomization method. Lastly, the numbers of subjects in the 4 studies were fairly different (100, 440, 149, 1, and 623, respectively). It was considered that there was a potential bias, which needs more high-quality examinations.

In conclusion, this meta-analysis revealed no significant association between preoperative MRI and surgical outcomes, which included mastectomy rate, breast conservation rate, and re-excision rate. This study also suggested that the use of preoperative MRI should be selective and should not be used as a routine preoperative examination based on this result. But due to the limited randomized trials, more randomized trials are needed for further study.

\section{Conflict of interest}

The authors declare no conflict of interest.

\section{References}

1. Guo R, Lu G, Qin B, Fei B. Ultrasound imaging technologies for breast cancer detection and management: a review. Ultrasound Med Biol 2018; 44: 37-70.

2. Rzymski P, Opala T. Elastography as a new diagnostic tool to detect breast cancer - evaluation of research and clinical applications. Prz Menopauzalny 2011; 5: 357-62.

3. Rzymski P, Wysocki PJ, Kycler W, Opala T. Correlation between insulin resistance and breast elasticity heterogeneity measured by shear wave elastography in premenopausal women - a pilot study. Arch Med Sci 2011; 6: 1017-22.

4. Rzymski P, Opala T. Morphological analysis of breast cystic lesions in shear wave elastography. Współcz Onkol 2011; 4: 208-12.

5. Leithner D, Wengert GJ, Helbich TH, et al. Clinical role of breast MRI now and going forward. Clin Radiol 2017; 73: 700-14.

6. Raghavendra AS, Tripathy D. How does MR imaging help care for the breast cancer patient? Perspective of a medical oncologist. Magn Reson Imaging Clin N Am 2018; 26: 289-93.

7. Ray KM, Hayward JH, Joe BN. Role of MR imaging for the locoregional staging of breast cancer. Magn Reson Imaging Clin N Am 2018; 26: 191-205.

8. Le-Petross HT, Stafford RJ. The need for MRI before breast-conserving surgery. Curr Breast Cancer Rep 2009; 1: 98-103.

9. Arnaout A, Catley C, Booth CM, et al. Use of preoperative magnetic resonance imaging for breast cancer: a Canadian population-based study. JAMA Oncol 2015; 1: 1238-50.

10. Gonzalez V, Sandelin K, Karlsson A, et al. Preoperative $M R I$ of the breast (POMB) influences primary treatment in breast cancer: a prospective, randomized, multicenter study. World J Surg 2014; 38: 1685-93.

11. Turnbull L, Brown S, Harvey I, et al. Comparative effectiveness of MRI in breast cancer (COMICE) trial: a randomised controlled trial. Lancet 2010; 375: 563-71.

12. Brück N, Koskivuo I, Boström P, Saunavaara J, Aaltonen R, Parkkola R. Preoperative magnetic resonance imaging in patients with stage I invasive ductal breast cancer: a prospective randomized study. Scand J Surg 2018; 107: 14-22.

13. Katipamula R, Degnim AC, Hoskin T, et al. Trends in mastectomy rates at the Mayo Clinic Rochester: effect of surgical year and preoperative magnetic resonance imaging. J Clin Oncol 2009; 27: 4082-8.

14. Houssami N, Turner RM, Morrow M. Meta-analysis of pre-operative magnetic resonance imaging (MRI) and surgical treatment for breast cancer. Breast Cancer Res Treat 2017; 165: 273-83.

15. Xu R, Tang P, Li C. Association between pre-operative magnetic resonance imaging (MRI) and surgical outcomes in breast cancer: not yet determined. Breast Cancer Res Treat 2019; 173: 749-50.

16. Peters NH, van Esser S, van den Bosch MA, et al. Preoperative MRI and surgical management in patients with nonpalpable breast cancer: The MONET - randomised controlled trial. Eur J Cancer 2011; 47: 879-86.

17. Bedrosian I, Mick R, Orel SG, et al. Changes in the surgical management of patients with breast carcinoma based on preoperative magnetic resonance imaging. Cancer 2003; 98: 468-73.

18. Liberman L, Morris EA, Dershaw DD, Abramson AF, Tan LK. MR imaging of the ipsilateral breast in women with 
percutaneously proven breast cancer. AJR Am J Roentgenol 2003; 180: 901-10.

19. Schelfout K, Goethem MV, Kersschot E, et al. Contrast-enhanced MR imaging of breast lesions and effect on treatment. Eur J Surg Oncol 2004; 30: 501-7.

20. Schnall MD, Blume J, Bluemke DA, et al. MRI detection of distinct incidental cancer in women with primary breast cancer studied in IBMC 6883. J Surg Oncol 2005; 92: 32-8.

21. Tan JE, Orel SG, Schnall MD, Schultz DJ, Solin LJ. Role of magnetic resonance imaging and magnetic resonance imaging--guided surgery in the evaluation of patients with early-stage breast cancer for breast conservation treatment. Am J Clin Oncol 1999; 22: 414-8.

22. Tillman GF. Effect of breast magnetic resonance imaging on the clinical management of women with early-stage breast carcinoma. J Clin Oncol 2002; 20: 3413-23.

23. Hollingsworth AB, Stough RG, O'Dell CA, Brekke CE. Breast magnetic resonance imaging for preoperative locoregional staging. Am J Surg 2008; 196: 389-97.

24. Sardanelli F. Sensitivity of MRI versus mammography for detecting foci of multifocal, multicentric breast cancer in fatty and dense breasts using the whole-breast pathologic examination as a gold standard. Am J Roentgenol 2004; 183: 1149-57.

25. Hlawatsch A, Teifke A, Schmidt M, Thelen M. Preoperative assessment of breast cancer: sonography versus MR imaging. AJR Am J Roentgenol 2002; 179: 1493-501.

26. Bagley FH. The role of magnetic resonance imaging mammography in the surgical management of the Index Breast Cancer. Arch Surg 2004; 139: 380-3.

27. Houssami N, Ciatto S, Macaskill P, et al. Accuracy and surgical impact of magnetic resonance imaging in breast cancer staging: systematic review and meta-analysis in detection of multifocal and multicentric cancer. J Clin Oncol 2008; 26: 3248-58.

28. Adrada BE, Candelaria R, Rauch GM. MRI for the staging and evaluation of response to therapy in breast cancer. Top Magn Reson Imaging 2017; 26: 211-8.

29. Huszno J, Kołosza Z, Tęcza K, Pamuła-Piłat J, Mazur M, Grzybowska E. Comparison between NOD2 gene mutation carriers (3020insC) and non-carriers in breast cancer patients: a clinicopathological and survival analysis. Arch Med Sci Civil Dis 2018; 3: e10-5.

30. Hao JQ, Zhang QK, Zhou YX, Chen LH, Wu PF. Association between circulating leptin concentration and G-2548A gene polymorphism in patients with breast cancer: a meta-analysis. Arch Med Sci 2019; 15: 275-83.

31. Momtazi-Borojeni AA, Nik ME, Jaafari MR, Banach $M$, Sahebkar A. Effects of immunization against PCSK9 in an experimental model of breast cancer. Arch Med Sci 2019; 15: 570-9.

32. Koczkodaj P, Sulkowska U, Gotlib J, Mańczuk M. Breast cancer mortality trends in Europe among women in perimenopausal and postmenopausal age (45+). Arch Med Sci 2020; 16: 146-56.

33. Khademi F, Vaez H, Momtazi-Borojeni AA, Majnooni A, Banach M, Sahebkar A. Bacterial infections are associated with cardiovascular disease in Iran: a meta-analysis. Arch Med Sci 2019; 15: 902-11.

34. Hall GH, Guthrie KA, Turnbull LW. Magnetic resonance imaging appearances of the Mirena and GyneFix intra-uterine contraceptive devices: a report of two cases. Br J Fam Plann 2000; 26: 224-6.

35. Debra L. Practical considerations for the use of breast MRI for breast cancer evaluation in the predictive setting. Acad Radiol 2017; 24: 1447-50. 